Original

\title{
Hipogonadismo prolongado tras la suspensión de tratamiento hormonal en pacientes con cáncer de próstata
}

\author{
Federico I. Rodríguez-Rubio Cortadellas, Miguel E. Jiménez Romero, Delfín González Moreno, \\ José P. Novalbos Ruiz*, Sofía Garrido Insúa
}

Servicio de Urología. Hospital Universitario Puerto Real. Departamento de Cirugía. Universidad de Cádiz, España. *Área de Medicina Preventiva. Facultad de Medicina. Universidad de Cádiz, España.

\begin{abstract}
Resumen
Objetivos: Estudiar los niveles de LH, testosterona y PSA tras suspender el tratamiento prolongado con análogos LH-RH Material y Método: Se estudió la evolución hormonal de 29 pacientes a los que se les retiró el tratamiento. Los pacientes previamente habían seguido tratamiento con análogo LH-RH por más de un año y con $\mathrm{LH}<2 \mathrm{mUI} / \mathrm{mL}$ y testosterona $<2,8$ $\mathrm{ng} / \mathrm{mL}$. Se determinó mensualmente la LH, testosterona y PSA junto con valoración clínica. Se reinicia el tratamiento y el tiempo de seguimiento finaliza ante la presencia de progresión clínica y/o PSA $\geq 10 \mathrm{ng} / \mathrm{mL}$. Se realizó descripción de la cohorte, el cálculo de supervivencia mediante Kaplan-Meier y regresión de Cox.

Resultados: El tiempo medio sin tratamiento de la serie fue de 35 meses (IC 95\% 15,7-54,2 meses). El 17\% de los pacientes presentaron hipogonadismo prolongado (>24 meses). La recuperación del eje LH-T-PSA, cuando se produjo, siguió la secuencia esperada. Las variables que influyeron en el tiempo de recuperación del PSA fueron el PSA pretratamiento y la asociación de antiandrógeno.

Conclusiones: Tras retirar el tratamiento prolongado con análogos LH-RH la mayoría de los pacientes recuperan los niveles de LH-T-PSA si bien un subgrupo se mantienen hipogonádicos más de 24 meses.
\end{abstract}

Palabras clave: Cáncer de próstata. Supresión androgénica intermitente. Testosterona. Antígeno prostático específico. Estudio prospectivo.

\section{Prolonged hypogonadism after cessation of androgen deprivation therapy for prostate cancer}

\section{Abstract}

Objectives: To study the levels of LH, testosterone and PSA after suspending prolonged treatment with LH-RH analogs. Materials and Method: Hormonal evolution was studied in 29 patients from whom treatment had been withdrawn. The patients had previously been receiving treatment with $\mathrm{LH}-\mathrm{RH}$ analog for more than one year, and with $\mathrm{LH}<2 \mathrm{mUI} / \mathrm{mL}$ and testosterone $<2.8 \mathrm{ng} / \mathrm{mL}$. LH, testosterone and PSA were determined monthly, together with clinical assessment. The treatment was re-initiated and the period of monitoring ended before the presence of clinical progression and/or PSA $\geq 10$ $\mathrm{ng} / \mathrm{mL}$. The cohort was described and survival was calculated using Kaplan-Meier and Cox regression.

Results: The mean period of time without treatment for the series was 35 months (CI 95\%, 15.7-54.2 months). Prolonged hypogonadism (> 24 months) was presented by $17 \%$ of the patients. The recovery of the LH-T-PSA axis, when it occurred, followed the expected sequence. The variables that influenced the period of recovery of the PSA were the PSA pretreatment and the association of an antiandrogen.

Conclusions: After withdrawing the prolonged treatment with LH-RH analogs, most of the patients recovered the levels of LH-T-PSA, although a subgroup remained hypogonadic for more than 24 months.

Keywords: Prostate cancer. Intermittent androgen supression. Testosterone. Prostate-specific antigen. Prospective studies.

$\mathrm{E}$ n el año 1941 Huggins y Hodges establecieron la andrógeno dependencia del cáncer de próstata $(\mathrm{CaP})^{1}$. Desde entonces, la orquiectomía, los estrógenos y los análogos LH-RH se han utilizado para disminuir la testosterona (T) a niveles de castración.
Los análogos de la LH-RH, inducen castración médica al interferir con la secreción normal de la hormona luteinizante (LH), anulando el eje LH-T-próstata. Sus efectos están bien documentados, producen niveles de castración de $\mathrm{T}$ a los 10-21 días y de forma continuada mientras se repitan las dosis ${ }^{2}$. 
Aunque se cree que la castración médica es un fenómeno reversible, el tiempo en el que la $\mathrm{T}$ y $\mathrm{LH}$ retornaría a niveles normales o basales ha sido poco estudiado $^{3-5}$. Además, la importancia de la cinética de la $\mathrm{T}$ tras la supresión del tratamiento con análogos ha sido destacada en esquemas de tratamiento hormonal intermitente y de neoadyuvancia (la única forma de valorar en $\mathrm{CaP}$ ). Si el efecto del tratamiento hormonal es prolongado, mayor de lo esperado por su vida media, podría favorecerse la supervivencia bioquímica en los ensayos de tratamiento radical y neoadyuvancia ${ }^{3}$.

Por otro lado, sabemos que la monitorización de los pacientes con $\mathrm{CaP}$ en tratamiento, la realizamos habitualmente con el antígeno prostático específico (PSA) que es un marcador órgano específico, cuya expresión es fuertemente andrógeno dependiente ${ }^{6}$. Si en la supresión androgénica con análagos LH$\mathrm{RH}$, el control del PSA se consigue con la supresión previa de la T, y ésta a su vez con la supresión previa de la $\mathrm{LH}$, lo lógico sería que la eficacia de dicho tratamiento se valorara no solo mediante la determinación del PSA, sino también con la LH y la T que son objetivos primarios de la medicación.

Así, el consejo general durante la reunión anual de la EAU de 2006 llego a la conclusión de que la $\mathrm{T}$ es la diana de la terapia hormonal, pero hasta hace poco no se disponía de las herramientas adecuadas para determinar con precisión los niveles de T. Se habla de que tendríamos que centrarnos no sólo en el PSA, sino también tener en cuenta los niveles de $T^{7}$.

En el presente trabajo, estudiamos una cohorte de pacientes con CaP bien definidos, a los que tras un tiempo de tratamiento crónico con análogos LH-RH, se les ha suspendido dicho tratamiento $\mathrm{y}$ se monitorizaron parámetros hormonales, bioquímicos y hematológicos. El objetivo será estudiar la recuperación de la $\mathrm{LH}$, la $\mathrm{T}$ y PSA en pacientes con $\mathrm{CaP}$ que han tenido supresión androgénica prolongada y dilucidar que variables influyen en dicha recuperación.

\section{MATERIAL Y MÉTODO}

Estudio observacional prospectivo de pacientes con CaP en cualquier estadio clínico sometidos a tratamiento crónico con análogos LH-RH con/sin antiandrógenos en los que estudiamos la evolución androgénica tras la suspensión del tratamiento mediante monitorización analítica mensual.

Los criterios de inclusión fueron: supresión androgénica durante al menos 1 año, ausencia de tratamientos por CaP previo a la inclusión, presentar niveles de LH y $\mathrm{T}$ por debajo de la normalidad ( $\mathrm{LH}<2 \mathrm{mUI} / \mathrm{mL}$ y $\mathrm{T}<2,8 \mathrm{ng} / \mathrm{ml}$ ), ausencia de trastorno o medicación que pueda interferir con los niveles hormonales, control oncológico (PSA <1 $\mathrm{ng} / \mathrm{mL}$ ), buen estado general (Karnosky >80\%), aceptación y consentimiento del protocolo.

A los pacientes que cumplían los criterios se les realizó monitorización mensual que consistía en controles clínicos en el momento de la suspensión del tratamiento y determinación mensual de PSA, T, LH, indefinidos hasta que presentasen alguno de los criterios de reinicio de tratamiento. Se añadieron las pruebas complementarias habituales (hemograma, bioquímica, pruebas de imagen...) según los criterios oncológicos habituales.

Los criterios de reinicio de tratamiento fueron PSA igual o mayor de $10 \mathrm{ng} / \mathrm{ml}$, progresión clínica objetiva y/o por deseo del paciente. Por lo tanto, el periodo de seguimiento evaluado en este estudio comprende desde la retirada del tratamiento hormonal hasta el reinicio del tratamiento (criterios previamente definidos) o muerte/pérdida de seguimiento (Fig. 1).

Todos los pacientes fueron informados previamente a la inclusión en este estudio. El estudio fue aprobado por la Comisión Ética y de Ensayos Clínicos de nuestro Centro.

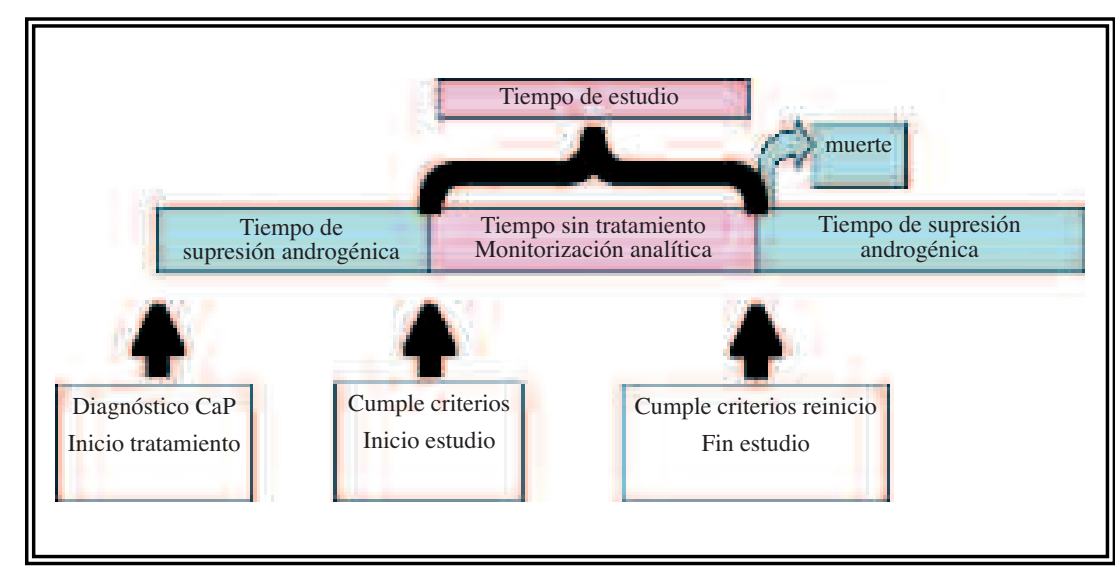

FIGURA 1: Se muestra gráficamente un paciente incluido en el estudio. El periodo de estudio comprende desde la suspensión del tratamiento hormonal hasta el reinicio o fallecimiento del paciente. 
La determinación de LH, T y PSA se realizó por inmunoensayo ECLIA Cobas. Los rangos de normalidad en varones propuestos por el laboratorio fueron para la $\mathrm{LH}$ de $2-8,6 \mathrm{mUI} / \mathrm{mL}$ y para la $\mathrm{T}$ de 2,8 $8 \mathrm{ng} / \mathrm{ml}$

Se definió la supervivencia como el tiempo transcurrido desde la suspensión del tratamiento (tiempo sin tratamiento de figura 1) y la última revisión o la recuperación de LH, T y PSA. Las medianas de supervivencia se estudiaron por el método de KaplanMeier. Se establecieron los siguientes puntos de corte: $\mathrm{LH} \geq 2 ; \mathrm{T} \geq 0,2 \mathrm{y} \geq 0,5$; PSA de $\geq 1, \geq 4 \mathrm{y} \geq 10$. Para evaluar que variables independientes permiten estimar, en función del tiempo, la probabilidad de reinicio de tratamiento o recuperación de valores de LH, T y PSA se han obtenido un modelo de riesgo proporcional de Cox para los diferentes puntos de corte.

\section{RESULTADOS}

De todos los pacientes inicialmente seleccionados 29 cumplieron los criterios de inclusión. Las características de los estos pacientes se muestran en la Tabla 1. 3 pacientes fallecieron durante el seguimiento por causas no relacionadas con el CaP. No hubo ninguna pérdida de seguimiento en el resto de pacientes durante el estudio.

Tabla 1. Características de los 29 pacientes estudiados

\begin{tabular}{lc}
\hline Variable & Número (\%) \\
\hline Número de pacientes & 29 \\
Edad (media) & 72,5 \\
Duración del tratamiento hormonal (meses) & 33,6 \\
Tipo Tratamiento & \\
Análogos + Antiandrógeno & $27(93)$ \\
Análogos monoterapia & $2(7)$ \\
Puntuación Gleason en Biopsia & \\
$\quad<4$ & $3(10)$ \\
$\quad 4-6$ & $22(76)$ \\
$\quad>7$ & $4(14)$ \\
PSA pretratamiento (ng/ml) & \\
$\quad$ Media & 42,2 \\
$\quad$ Rediana & 19,2 \\
$\quad$ aango & $3,2-312,5$ \\
Tacto rectal & $17(58)$ \\
$\quad$ Anormal & $12(42)$ \\
Metástasis en diagnóstico & $3(10,3)$ \\
\hline
\end{tabular}

21 pacientes $(72,4 \%)$ reiniciaron tratamiento $\mathrm{y}$ en $8(27,6 \%)$ no fue necesario reiniciar nuevamente el tratamiento hormonal al cierre del estudio.

De los 21 pacientes que requirieron reinicio de tratamiento, $13(61,9 \%)$ lo hicieron por elevación de PSA ( $\geq 10 \mathrm{ng} / \mathrm{ml}), 5(23,8 \%)$ por criterios clínicos y 3 $(14,2 \%)$ por enfermedades asociadas que presentaron o ya padecían durante el transcurso del estudio. El tiempo medio de seguimiento hasta el reinicio de tratamiento en estos pacientes fue de 23,8 meses.

De los 8 que nunca reiniciaron tratamiento, 3 fallecieron por causas ajenas al CaP durante el seguimiento (tiempo medio de seguimiento de 14 meses). 5 pacientes no han reiniciado el tratamiento (tiempo medio de seguimiento de 64,5 meses $(5,3$ años).

El tiempo de recuperación de la $\mathrm{LH}$ fue 6,3 meses (rango: 1-63) y el tiempo de recuperación de la $\mathrm{T}$ fue de 8,2 meses (rango: 1-57).

La mediana de tiempo de los periodos sin tratamiento de toda nuestra serie fue de 35 meses (IC 95\% 15,7-54,2 meses) y en los pacientes con tratamiento combinado con flutamida o bicalutamida la mediana fue mayor (IC 95\% 25,2-50,7 meses y 0-73 meses respectivamente) Tabla 2 .

El estado de la $\mathrm{T}$ al año y a los 2 años se muestra en la Tabla 3. Distinguimos pacientes que no superan el nivel de castración $(<0,5 \mathrm{ng} / \mathrm{ml})$ de los pacientes hipogonádicos, es decir, que no alcanzan los niveles de normalidad ( $<2,8 \mathrm{mUI} / \mathrm{mL})$. $\mathrm{Al}$ año sin tratamiento $10(34 \%)$ pacientes mantienen niveles de castración y 16 (55\%) son hipogonádicos. A los 2 años 5 (17\%) de los pacientes mantienen niveles de castración y 11 (38\%) son hipogonádicos. A ese seguimiento todos excepto uno habían recuperado los niveles de LH.

En la Tabla 4 mostramos los 5 pacientes que mantuvieron una situación de castración muy prolongada a pesar de haber sido suspendido el tratamiento (más de 24 meses). Todos excepto uno habían recuperado la $\mathrm{LH}$ e incluso esta en algunos casos superaba de la normalidad. Al final de seguimiento de estos 5 pacientes el PSA se mantuvo en su nadir y en ninguno existía signos clínicos de progresión.

Cuando estudiamos que variables permiten estimar, en función del tiempo, la probabilidad de recuperación de PSA ( $\mathrm{PSA} \geq 4 \mathrm{ng} / \mathrm{ml}$; PSA $\geq 10 \mathrm{ng} / \mathrm{ml}$ ) encontramos que influyen de forma significativa en este tanto el PSA pretratamiento, como la asociación de antiandrógeno al análogo LH-RH (Tabla 5); en el 
Tabla 2. Estimación del tiempo de supervivencia (meses) sin necesidad de reinicio de tratamiento.

\begin{tabular}{|c|c|c|c|c|c|c|c|c|}
\hline \multirow[b]{3}{*}{ Antiandrógeno } & \multicolumn{4}{|c|}{ Media (meses) } & \multicolumn{4}{|c|}{ Mediana } \\
\hline & \multirow[b]{2}{*}{ Tiempo } & \multicolumn{3}{|c|}{ IC 95\% } & \multirow[b]{2}{*}{ Estimación } & \multirow[b]{2}{*}{$\begin{array}{c}\text { Error } \\
\text { típico }\end{array}$} & \multicolumn{2}{|c|}{ IC 95\% } \\
\hline & & $\begin{array}{l}\text { Error } \\
\text { típico }\end{array}$ & $\begin{array}{l}\text { Limite } \\
\text { inferior }\end{array}$ & $\begin{array}{c}\text { Limite } \\
\text { superior }\end{array}$ & & & $\begin{array}{l}\text { Limite } \\
\text { inferior }\end{array}$ & $\begin{array}{c}\text { Limite } \\
\text { superior }\end{array}$ \\
\hline Ninguno & 10,00 &, 00 & 10,00 & 10,00 & 10,00 & - & - & - \\
\hline Bicalutamida & 32,52 & 7,32 & 18,15 & 46,88 & 34,00 & 20,14 &, 00 & 73,49 \\
\hline $\begin{array}{l}\text { Acetato } \\
\text { ciproterona }\end{array}$ & 5,00 & ,00 & 5,00 & 5,00 & 5,00 & - & - & - \\
\hline Global & 34,94 & 4,31 & 26,49 & 43,39 & 35,00 & 9,80 & 15,79 & 54,20 \\
\hline
\end{tabular}

Tabla 3. Situación del eje LH-T-PSA al año y dos 2 años de los 29 pacientes incluidos en el estudio. Puntos de corte para el PSA de 1,4 y $10 \mathrm{ng} / \mathrm{mL}$. Porcentaje de pacientes con niveles de castración $(\mathrm{T}<0,5 \mathrm{ng} / \mathrm{ml})$ y de pacientes hipogonádicos (niveles de $\mathrm{T}$ por debajo del rango de la normalidad: $2,8 \mathrm{ng} / \mathrm{ml}$ ) para ese mismo tiempo de seguimiento.

\begin{tabular}{lcc}
\hline & 12 meses & 24 meses \\
\hline $\mathrm{N}^{\circ}$ pacientes con PSA < $\left.1 \%\right)$ & $14(48,2 \%)$ & $9(31 \%)$ \\
$\mathrm{N}^{\circ}$ pacientes con PSA < $4(\%)$ & $18(62 \%)$ & $11(37,9 \%)$ \\
$\mathrm{N}^{\circ}$ pacientes con PSA < $10(\%)$ & $19(65,5 \%)$ & $14(48,2 \%)$ \\
$\mathrm{N}^{\circ}$ pacientes con niveles de & $10(34,5 \%)$ & $5(17,2 \%)$ \\
castración (\%) & $16(55 \%)$ & $11(37,9 \%)$ \\
$\mathrm{N}^{\circ}$ pacientes hipogonádicos $(\%)$ & $16 \%)$ & $1(3,44 \%)$ \\
$\mathrm{N}^{\circ}$ pacientes con LH < 2 (\%) & $1(3,44 \%)$ & \\
\hline
\end{tabular}

caso de la flutamida el efecto es claramente protector $(\operatorname{Exp}(\beta): 0,10)$, mientras que para la bicalutamida el bajo número de pacientes y la mayor variabilidad en las cifras de supervivencia no nos permiten confirmar este efecto (sig 0,058). Utilizando el mismo modelo, ninguna variable fue claramente influyente en relación con la recuperación de la $\mathrm{T}$ y la $\mathrm{LH}$.

En la curva de supervivencia de muestra (Fig. 1) podemos apreciar que a igualdad de valores de PSApretatamiento los pacientes tratados con flutamida y bicalutamida presentan un mayor tiempo libre de reinicio de tratamiento frente a aquellos tratados con acetato de ciproterona o sin tratamiento.

\section{DISCUSIÓN}

Desde 1941 la supresión androgénica es una opción en el tratamiento del CaP. Habitualmente la supresión androgénica es permanente y se consigue a través de la castración quirúrgica o bien con tratamiento con análogos de la LH-RH de forma continua. El nivel óptimo y clásico de castración permanente de la $\mathrm{T}$ para un buen control oncológico ha sido de $0,5 \mathrm{ng} / \mathrm{mL}$ en pacientes con tratamiento médico $^{8}$. Si la supresión androgénica se realiza con análogos LH-RH, la supresión constante de la T se realiza a través de la supresión previa de la $\mathrm{LH}$.

Tabla 4. Características de 5 pacientes con hipogonadismo muy prolongado a pesar de la suspensión de la supresión androgénica. Los niveles de T, LH y PSA corresponde al último mes de seguimiento. Obsérvese que aunque algunos superan el nivel de castración la $\mathrm{T}$ se mantiene muy por debajo de límite inferior de normalidad. Pacientes con $\mathrm{T}$ a nivel de castración* y pacientes que han superado nivel de castración pero sin alcanzar la normalidad**. Respecto a la LH todos menos uno alcanzaron los niveles de normalidad ${ }^{\#}$ y en 2 pacientes su nivel de LH fue superior al rango de normalidad $^{\# \# . ~}$

\begin{tabular}{ccccccc}
\hline Edad & $\begin{array}{c}\text { Meses tratamiento } \\
\text { análogos }\end{array}$ & $\begin{array}{c}\text { Meses hipogonadismo } \\
\text { post-tratamiento }\end{array}$ & Nivel de T & Nivel de LH & PSA \\
\hline 1 & 76 & 39 & 24 & $0,17^{*}$ & $13^{\# \#}$ & 0,10 \\
2 & 63 & 45 & 70 & $0,58^{* *}$ & 4,84 & 0,70 \\
3 & 78 & 60 & 57 & $0,05^{*}$ & 6,66 & 0,04 \\
4 & 69 & 24 & 65 & $0,72^{* *}$ & $8,72^{\# \#}$ & 0,72 \\
5 & 71 & 6 & 66 & $0,62^{* *}$ & $0,10^{\#}$ & 1,25 \\
\hline
\end{tabular}


Tabla 5. Modelo de regresión de Cox. Variables influyentes en la probabilidad de reinicio de tratamiento en función del tiempo (PSA $\geq 10 \mathrm{ng} / \mathrm{mL}$ )

\begin{tabular}{|c|c|c|c|c|c|c|c|c|}
\hline & \multirow[b]{2}{*}{ B } & \multirow[b]{2}{*}{ ET } & \multirow[b]{2}{*}{ Wald } & \multirow[b]{2}{*}{ G1 } & \multirow[b]{2}{*}{ Sig } & \multirow[b]{2}{*}{$\operatorname{Exp}$} & \multicolumn{2}{|c|}{$95 \%$ IC para Exp (B) } \\
\hline & & & & & & & Inferior & Superior \\
\hline PSA_ pretratamiento &, 014 & ,004 & 10,957 & 1 & ,001 & 1,014 & 1,006 & 1,023 \\
\hline Antiandrógeno & 11,291 & 3 & ,009 & & & & & \\
\hline Flutamida & $-2,221$ & 1,052 & 4,453 & 1 & ,035 &, 109 &, 014 & ,854 \\
\hline Acetato ciproterona & 2,795 & 1,691 & 2,730 & 1 & ,098 & 16,357 &, 594 & 450,115 \\
\hline
\end{tabular}

Hay pocos estudios sobre la cinética de la recuperación de LH y $\mathrm{T}$ tras el cese de la supresión androgénica en el CaP. La reversibilidad del tratamiento con análogos LH-RH se ha valorado en su aplicación por periodos de tiempo corto y en otras circunstancias (voluntarios y mujeres). Linde et al observaron en 8 varones sanos voluntarios tratados con análogos LH-RH ( $<10$ semanas) la recuperación tanto de LH como T en 4 semanas $^{9}$. Por otro lado, se sabe que la función ovárica se recupera en 8 semanas en mujeres jóvenes tratadas con análogos ${ }^{10}$.

En el caso del CaP, se han propuesto estrategias de supresión androgénica no permanente como la neoadyuvancia con radioterapia/cirugía ${ }^{11}$ o en el tratamiento hormonal intermitente ${ }^{12}$. Estas opciones de tratamiento permiten valorar la duración del efecto de la supresión androgénica cuando ya el tratamiento ha sido suspendido.

Oefelein ${ }^{5}$ estudió, en 13 pacientes con CaP tras una única inyección de agonista LH-RH trimestral (leuprolide o goserelina), la duración de la supresión androgénica y de los síntomas clínicos derivados de esta. Los niveles de castración persistieron en el $77 \%$ a los 6 meses y los síntomas de hipogonadismo persistieron una media de 13,6 meses. Egawa et al. ${ }^{13}$ estudiaron 10 pacientes con una única dosis de análogo LH-RH y observaron que el 83\% recuperaron los niveles normales de testosterona en 3 meses.

Pero hay menos información de la reversibilidad del eje si el tratamiento con análogos de la LH-RH se prolonga por más de 1 año. Hall et al. ${ }^{4}$ estudiaron 14 pacientes que recibieron un mínimo de 24 meses de tratamiento. En los primeros 6 meses no observaron cambios significativos y un año después de la retirada del tratamiento la media de los niveles de $\mathrm{T}$ permanecían por debajo de $1,11 \mathrm{ng} / \mathrm{ml}$ (su rango de normalidad 3-10 ng/ml) y 4 pacientes (29\%) permanecían en niveles de castración $(<0,5 \mathrm{ng} / \mathrm{ml})$.
Parece que de una lectura inicial de la literatura, la recuperación del eje LH-T-próstata puede depender del escenario en el que se aplique la supresión androgénica. Los jóvenes sanos pueden recuperase pronto mientras que tratamientos prolongados y en mayores, como ocurre en pacientes con $\mathrm{CaP}$, tardaría más tiempo dicha recuperación.

Sin embargo, como veremos más adelante, la obtención de conclusiones claras de los datos de los estudios que han abordado el tema se hace muy difícil ya que las referencias para la normalización hormonal son muy variadas. La definición de recuperación para unos es superar el nivel de castración y para otros es recuperar el rango de normalidad que además varía de un estudio a otro. Además, también varían de unos estudios a otros los tiempos de tratamiento y los tratamientos adicionales (radioterapia, cirugía, antiandrógenos...).

En nuestro análisis multivariante encontramos que tanto el PSA pretratamiento como la asociación de algún tipo de antiandrógeno periférico al análogo LH-RH influían en la recuperación del PSA y del reinicio del tratamiento. Estos datos coinciden con los derivados de un meta-análisis en pacientes con $\mathrm{CaP}$ en tratamiento hormonal intermitente ${ }^{12}$. Las variables que predijeron una mayor duración de los periodos sin tratamiento fueron el PSA inicial el PSA nadir y el tipo de tratamiento.

En el caso de la T, las variables que se han relacionado con la recuperación de la misma tras la suspensión del tratamiento son: el tiempo de tratamiento previo ${ }^{3,13}$, la edad ${ }^{3}$, la $\mathrm{T}$ previa al inicio del tratamiento ${ }^{14-16}$. Es muy difícil obtener conclusiones de los datos de estos estudios ya que la influencia de una variable varía desde el todo o nada en los distintos estudios. En nuestro caso, ninguna de las variables estudiadas demostró influir claramente en la recuperación de la T. Existen potenciales razones para estas discrepancias. Nuestra población corres- 
ponde a pacientes con tiempos de tratamientos muy prolongados y es posible que a partir del año de tratamiento no sea posible la recuperación gonadal en buen número de ellos ${ }^{17,18}$ y así la duración del tratamiento no influye en este grupo de pacientes. Sólo en aquellos estudios donde se incluye población con deprivación androgénica variada respecto al tiempo de tratamiento (tanto en ciclos cortos como prolongados) se ha observado como el tiempo de tratamiento influye en la recuperación gonadal ${ }^{3,13}$. La mayoría de los estudios corresponden a pacientes que se han tratado con radioterapia y deprivación androgénica transitoria. Esta población difiere de la muestra donde habitualmente son más jóvenes al tener opción de tratamiento con intención curativa y además por el hecho demostrado de que la radioterapia produce daño gonadal adicional ${ }^{19,20}$.

Aunque es posible que los jóvenes tengan un perfil hormonal previo más favorable para la recuperación temprana ${ }^{9,10}$, también es verdad que las poblaciones estudiadas por nosotros y otros se encuentran concentradas en rangos de edad avanzada y puede ser la razón de que la edad no influya en el escenario del CaP.

Algunos estudios han mostrado que la situación hormonal basal influye en la recuperación de los niveles de T. La T basal baja está relaciona con recuperación más lenta ${ }^{14-16}$. Murthy et al. ${ }^{21}$ precisaron más al observar que tanto la LH y FSH basal como la relación LH/FSH bajas predice la recuperación rápida. El diseño de nuestro estudio, en el que la inclusión de pacientes correspondía a pacientes ya en deprivación androgénica, no nos permitió comprobar esta influencia hormonal basal.

Una observación interesante de este estudio es la persistencia muy prolongada de la castración en algunos pacientes tras la suspensión del tratamiento. El hipogonadismo prolongado (no castrados pero $\mathrm{T}$ por debajo de la normalidad) ha sido descrito previamente de forma anecdótica en estudios con otros objetivos. Este estudio es el primero que describe una situación de castración (menos de 0,5 ng/mL) en tiempos tan prolongados. Hemos observado castración prolongado sin tratamiento (más de 2 años en 5 pacientes - 17\%) y tres de estos llegaron a superar los 5 años de castración. Las razones de la supresión gonadal en 4 fueron claramente testiculares en tanto que la LH se normalizó previamente e incluso a veces con valores supranormales. En relación con el PSA, como era de esperar en una situa- ción de hipogonadismo, se mantuvo en su nadir y ninguno presentó signos clínicos de progresión, hasta el final del seguimiento.

¿Es posible la castración prolongada sin tratamiento continuo? La supresión persistente de $\mathrm{T}$ tras la administración de estrógenos ha sido observada por otros estudios ${ }^{22-24}$. En el caso de supresión androgénica mediante análogos LH-RH la posibilidad de hipogonadismo muy prolongado ya fue señalado por Stevens ${ }^{25}$ que observa que durante 12 meses los pacientes presentaban sintomas de hipogonadismo y ya entonces advertía de que la acción exclusivamente aguda de los análogos LH-RH no se cumplía siempre. Nejat et al. ${ }^{3}$ describen que la ausencia de normalización (alcanzar rango de normalidad) en 5 de 7 (71\%) pacientes que previamente tuvieron supresión androgénica más de 24 meses. Hall et al. ${ }^{4}$ observaron en su estudio, que la $\mathrm{T}$ no superaba los niveles de castración en 4 de 14 $(28,6 \%)$ al año tras neoadyuvancia (3-6 meses) y radioterapia. En estos pacientes la supresión constante de T se asociaba a la supresión constante de LH (hipogonadismo hipofisario). Nosotros a los 12 meses y 24 meses teníamos $10(34,5 \%)$ y $5(17 \%)$ en castración y $16(55 \%)$ y 11 (38\%) en situación de hipogonadismo respectivamente. Además, observamos que a más de 2 años de castración 4 de 5 pacientes la persistencia de castración se asociaba a LH normal o alta por lo que se trata de un hipogonadismo primitivo. Shaidi et al. coinciden con nosotros al comunicar que en 2 pacientes con más de 2 años de castración sin tratamiento se asociaban a LH elevada ${ }^{26}$.

La potencial recuperación de la LH tras tratamiento prolongado con análogos LH-RH en pacientes con CaP está muy poco estudiada en la literatura. Sabemos que, cuando se inicia el tratamiento, la LH se incrementa causando el "fenómeno flare" y posteriormente baja a niveles indetectables ${ }^{27,28}$. La eventual suspensión del tratamiento debería llevar a una recuperación progresiva. Egawa et al. ${ }^{13}$ y Hall et al. ${ }^{4}$ documentaron que la $\mathrm{LH}$ se recupera de forma paralela a la T en la mayoría de sus 38 y 14 pacientes respectivamente en tratamiento durante tiempo muy prolongado. Por otro lado se ha sugerido de un efecto rebote-elevación paradójica- de las hormonas hipofisarias (LH y FSH $)^{9,21}$. La elevación prolongada por arriba de los valores normales de la $\mathrm{LH}$, una vez finalizado el tratamiento sugiere un daño testicular provocado por el tratamiento prolongado con análo- 
go LH-RH u otras causas como la radioterapia. La acción de los análogos LH-RH sobre las células de Leydig y de Sertoli se ha estudiado ${ }^{18,29}$.

En nuestro estudio la LH se recuperó en general de forma paralela a la $\mathrm{T}$ en todos los pacientes. El comportamiento de la LH, entre los 5 pacientes con más de 2 años sin recuperación de la T, fue muy variado: un paciente no recupera la $\mathrm{LH}$ lo que sugiere hipofisectomía química, dos pacientes presentaron niveles supranormales lo que sugiere daño testicular y sobre-estimulación de hipófisis-hipergonadismo primitivo e hipergonadotrópico- y en dos pacientes la LH se recupera a niveles dentro de la normalidad por lo que se trataría de hipogonadismo primitivo normagonadotrópico.

En estos pacientes añosos y con tratamiento prolongado con análogos, no sabemos actualmente si la recuperación definitiva de la LH se alcanza al llegar al rango de normalidad o si existe un nivel determinado inferior al límite de normalidad que estimula adecuadamente a la gónada para que secrete a su vez T.

A nuestro juicio el presente estudio presenta algunas limitaciones como es la ausencia de datos de la situación basal hormonal (LH y T). Aunque en su ausencia hemos podido comprobar la cinética de recuperación no hemos podido incluir estas variables tal como lo han hecho otros autores. También la medición de índice de masa corporal, que en ningún estudio de los recogidos lo utiliza, hubiera sido interesante en tanto que puede influir en el metabolismo hormonal.

De este tipo de estudio donde se destaca lo prolongado de la recuperación del eje hipófisis-gónadapróstata derivan una serie de implicaciones. Los diseños y en la interpretación de esquemas de tratamiento neoadyuvante o adyuvante que incluyan análogos LH-RH tienen que considerar el tiempo de recuperación de los niveles de $\mathrm{T}$. Los niveles de $\mathrm{T}$ deben ser medidos porque de estos niveles va a depender el PSA, que es la variable final que define la progresión bioquímica y tras esta el tiempo a la progresión clínica. Estos datos pueden explicar en parte, los resultados recientes de ensayos que combinando radiación y tratamiento hormonal, donde este tratamiento combinado tiene un mejor control del PSA que cuando sólo se utiliza la radiación.

Los niveles de $\mathrm{T}$ pueden tener también importantes implicaciones en el seguimiento de ensayos de tratamiento hormonal intermitente.
Nuestros datos apoyan el trabajo de Oefelein ${ }^{30}$ que sugiere la posibilidad de ajuste de dosis en el tratamiento con análogos LH-RH si monitorizamos los niveles de T. Así la pauta de tratamiento puede variar según el tiempo de recuperación de los niveles de $\mathrm{T}$ con menos inyecciones de análogos LH-RH implicaciones económicas muy importantes.

En conclusión, el presente trabajo aporta información sobre el efecto que tiene el tratamiento de supresión androgénica cuando se recibe de forma crónica sobre el eje hipófisis-gónada-próstata. Además destacamos que algunos pacientes pueden presentar situación de hipogonadismos o castración muy prolongados a pesar de haber suspendido el tratamiento. Actualmente se precisan más estudios con criterios bien definidos para definir completamente el patrón de recuperación hormonal tras un periodo de castración.

\section{REFERENCIAS}

1. Huggins C y Hodges C: Studies on prostatic cancer: the effect of castration of estrogen and androgen injection on serum phosphatases in metastatic carcinoma of the prostate. Cancer Res 1941;1:293.

2. Kuhn JM, Abourachid H, Brucher P, Doutres JC, Fretin J, Jaupitre A et al: A randomized comparison of the clinical hormonal effects of two GnRh agonist in patients with prostate cancer. Eur Urol 1997;32(4):397-403.

3. Nejat RJ, Rashid HH, Bagiella E, Katz AE, Benson MC. A prospective analysis of time to normalization of serum testosterone after withdrawal of androgen deprivation therapy. J Urol 2000; 164(6):1891-1894.

4. Hall MC, Fritzsch RJ, Sagalowsky AI, Ahrens A, Petty B, Roehrborn CG. Prospective determination of the hormonal response after cessation of luteinizing hormone-releasing hormone agonist treatment in patients with prostate cancer. Urology 1999;53:898-902.

5. Oefelein MG: Time to normalization of serum testosterone after 3-month luteinizing hormone-releasing hormone agonist administered in the neoadjuvant setting: implications for dosing schedule and neoadjuvant study consideration. J Urol 1998; 160:1685-1688.

6. Henttu P, Liao SS y Vihko P: Androgens up-regulate the human prostate-specific antigen messenger ribonucleic acid (mRNA), but down-regulate the prostatic acid phosphatase mRNA in the LNCaP cell line. Endocrinology 1992;130:766-772.

7. Calais Da Silva FM, Calais Da Silva F, Bono A, et al. Phase III intermittent MAB vs continuous MAB. J Clin Oncol 2006; 24: 220 (abstract no.4513).

8. Oefelein MG, Feng A, Scolieri MJ, Ricchiutti D, Resnick MI Reassessment of the definition of castrate levels of testosterone: implications for clinical decision making. Urology. 2000; 56(6): 1021-1024.

9. Linde R, Doelle GC, Alexander N, Kirchner F, Vale W, Rivier J, Rabin D: Reversible inhibition of testicular steroidogenesis and spermatogenesis by a potent gonadotrophin-releasing hormone agonist in normal men. N Eng J Med 1981;305(12) 663-667.

10. Comite F, Cutler GB Jr, Rivier J, Vale WW, Loriaux DL, Crowley WF Jr: Short-term treatment of idiopathic precocious puberty with a long-acting analogue of luteinizing hormone-releasing hormone. A preliminary report. N Engl J Med 1981;305:1546-1550. 
11. Bruchovsky N, Klotz L, Crook J, Malone S, Ludgate C, Morris WJ et al: Final results of the Canadian Prospective phase II of intermittent androgen suppression for men in biochemical recurrencia after radiotherapy for locally advanced prostare cancer. Cancer 2006;107:389-395.

12. Shaw GH, Wilson P, Cuzick J, Prowse DM, Goldenberg SL, Spry NA et al: International study into the use of intermittent hormone therapy in the treatment of carcinoma of the prostate: a meta-analysis of 1446 patients. BJU Int 2007;99:1056-1065.

13. Egawa S, Okusa H, Matsumoto K, Suyama K, Baba S: Changes in prostate-specific antigen and hormone levels following withdrawal of prolonged androgen ablation for prostate cancer. Prostate Cancer Prostatic Dis. 2003;(3):245-249.

14. Pickles T, Agranovich A, Berthelet E, Duncan GG, Keyes M, Kwan W et al: Testosterone recovery following prolonged adjuvant androgen ablation for prostate carcinoma. Cancer 2002; 94 (2):362-367.

15. Padula GD, Zelefsky MJ, Venkatraman ES, Fuks Z, Lee HJ, Natale L et al: Normalization of serum testosterone levels in patients treated with neoadjuvant hormonal therapy and threedimensional conformal radiotherapy for prostate cancer. Int Radiat Oncol Biol Phys 2002;52 (2):439-443.

16. Gulley JL, Figg WD, Steinberg SM, Carter J, Sartor O, Higano $\mathrm{CS}$ et al: A prospective analysis of the time to normalization of serum androgens following 6 months of androgen deprivation therapy in patients on a randomized phase II clinia trial using limited hormonal therapy. J Urol 2005;173:1567-1571.

17. Giberti C, Barreca T, Martorana G, Truini M, Franceschini R, Rolandi $\mathrm{E}$ et al: Hormonal pattern and testicular histology in patients with prostatic cancer after long-term treatment with a gonadotropin-releasing hormone agonist analogue. Eur Urol. 1988;15(1-2):125-127.

18. Kisman OK, de Voogt HJ, Baak JA: Reversibility of the effect of LHRH agonists and other antiandrogenic hormones on the testis: a histomorphometric study. Eur Urol 1990; 18 (4):299-301.

19. Izard MA: Leydig cell function and radiation: a review of the literature. Radiother Oncol 1995;34 (1):1-8.

20. Shapiro E, Kinsella TJ, Makuch RW, Fraass BA, Glatstein E, Rosenberg SA et al: Effects of fractionated irradiation of endocrine aspects of testicular function. J Clin Oncol. 1985;(9): 1232-1239.

21. Murthy V, Norman AR, Shahidi M, Parker CC, Horwich A, Huddart RA et al: Recovery of serum testosterone after neoadjuvant androgen deprivation therapy and radical radiotherapy in localized prostate cancer. BJU Int 2006;(3):476-479.
22. Robinson MR, Thomas BS: Effect of hormonal therapy on plasma testosterone levels in prostatic carcinoma. Br Med J 1971;4 (5784):391-394.

23. Tomic R, Bergman B. Hormonal effects of cessation of estrogen treatment for prostatic carcinoma. J Urol. 1987;138(4):801-805.

24. Zelefsky MJ, Leibel SA, Gaudin PB, Kutcher GJ, Fleshner NE, Venkatramen ES et al: Dose escalation with three-dimensionalconformed radiation therapy affectsthe outcome in prostate cancer. Int J Radiat Oncol Biol Phys 1998;41(3):491-500.

25. Stevens MJ, Bell DR, Blome SA y Begbie SD: Prolonged hypogonadism after pulsed gonadotropin-releasing hormone agonist for prostate cancer. Lancet 1994;344(8917):274-275.

26. Shahidi M, Norman AR, Gadd J, Huddart RA, Horwich A Dearnaley DP: Recovery of serum testosterone, LH and FSH levels following neoadjuvant hormone cytoreduction and radical radiotherapy in localized prostate cancer. Clin Oncol (R Coll Radiol) 2001;13(4):291-295.

27. Crawford ED, Eisenberger MA, McLeod DG, Spaulding JT, Benson R, Dorr FA et al: A controlled trialof leuprolide with and without flutamide in prostatic carcinoma. N Engl J Med 1989; 17;321(7):419-424.

28. Pavone-Macaluso M, de Voogt HJ, Viggiano G, Barasolo E, Lardennois B, de Pauw $\mathrm{M}$ et al: Comparison of diethylstilbestrol, cyproterone acetate and medroxyprogesterone acetate in the treatment of advanced prostatic cancer. Final analysis of a randomized phase III trial of the European Organization for Research on Treatment of Cancer Urological Group. J Urol 1986;136(3):624-631.

29. Huhtaniemi I, Nikula H, Parvinen M, Rannikko S: Histological and functional changes of the testis tissue during GnRH agonist treatment of prostatic cancer. Am J Clin Oncol 1988;11 Suppl 1:S11-15.

30. Oefelein MG: Serum testosterone-based luteinizing hormonereleasing hormone agonist redosing schedule for chronic androgen ablation: a phase I assessment. Urology 1999;54(4): 694-699.

Correspondencia autor: Dr. Federico I. Rodríguez-Rubio Cortadellas Servicio de Urología. Hospital Universitario Puerto Real Carretera Nacional IV, Km 665 - 11510 Puerto Real, Cádiz Tel.: 956005000 E-mail autor: frubio@pulso.com Información artículo: Original Trabajo recibido: febrero 2009

Trabajo aceptado: abril 2009 